\title{
A New Test of Artificial Intelligence: Should the Media Industry Be Afraid?
}

\author{
K. Horska \\ Institute of Journalism at Taras Shevchenko National University of Kyiv, Ukraine \\ Corresponding author. E-mail: Pravo-media@ukr.net
}

Paper received 30.05.20; Accepted for publication 16.06.20.

\begin{abstract}
https://doi.org/10.31174/SEND-HS2020-231VIII39-06
\end{abstract}
\begin{abstract}
Artificial Intelligence (AI) technologies assertively reveal their powerful potential in the media completely changing the idea of what the media production can be soon. Tools for editorial processes optimization and production costs reduction are offered to the media industry that is surviving the crisis of business models. Impressive results demonstrated by the products generated using AI force us to not only revise fundamental principles of the industry's work but also call into question the society's need for profession of a journalist. The presented study is intended to review examples of AI integration in the media and identify benefits and threats of those innovations from the standpoint of journalists themselves, as well as assess how dramatically those processes can change the balance of forces of humans vs AI.
\end{abstract}

Keywords: artificial intelligence (AI), media industry, digital journalism, fake news, data journalism.

Introduction. AI technologies rapidly penetrated in various areas of human activities striking imagination with their seemingly limitless possibilities and at the same time raising concerns in connection with the threatening prospect of complete substitute of human labor. In the media industry, AI was able to show its full potential by demonstrating high-quality content, an unprecedented degree of personalization, and effective interaction with the audience. AI has already come to the journalism, and the media simply face the dilemma of whether to respond to the challenges and adopt to the new reality or fight with "windmills" trying to stop the upcoming progress of innovations and promoting the model of journalism as a "people to people" activity.

Journalism has already faced many serious challenges. The surge in citizen journalism and the boom in user-generated content was also seen as a sign of the imminent death of journalism. However, it is still alive, and recently, under the onslaught of fakes and information propaganda, researchers have revisited discussions about the need to return to professional journalism. It is obvious that in the new situation, professionalism should both be based on experience and focus on up-to-date trends, and media's capability to utilize new technologies for their benefit.

Objective of the study. In this article, we attempt to identify trends in AI development, which directly influence journalist activities and the media market, and evaluate how grounded are the concerns of the industry experts with regard to this influence, especially as to retaining journalist jobs.

Overview of studies on the topic. It is obvious that the journalism will never be the same. The report for 2019 published by the European Broadcasting Union states that the $\mathrm{AI}$ is an expensive, however, necessary investment for the media. Experts say that journalists will continue to be in demand in the profession "especially for judgment skills", they admit that "almost all journalist roles will have to change" [1].

Other forecasts concerning journalists retaining their jobs are not so optimistic [2]. However, we cannot state that the declined demand for human resources in the media sector is directly caused by the progress in information and communication technologies and automated production and dissemination of media products. The worldwide crisis survived by the media is of a comprehensive nature.
Among its reasons, researchers indicate growth of the number of competitors in this segment with the advent of new media players - search systems, news aggregators [3, 4], active audience generating the content themselves, inefficiency of traditional models of media products monetization, rethought communications between journalists and automated systems [5], the need to understand the laws of digital journalism [6].

When speaking of the evaluation of the extent of AI implementation in journalism $[7,8]$, we need to understand whether AI can become a media partner and not a competitor and be used effectively to increase its effectiveness. Nowadays, it is a challenge, which is equally faced by news websites [9], TV and radio broadcasting companies [10].

Materials and methods. In the course of our study, we analyzed examples of AI implementation in the media by most significant media market players and highlighted the areas where their experience was most successful. Our analysis deliberately does not touch upon tools of social networks, which are just actively using AI achievements to analyze the content, recognize faces and images and for many other purposes. The focus is on the innovations used by the media on their own platforms.

In parallel, 19 semi-structured interviews were carried out with representatives of the media industry in order to find out how organically practices of using AI were integrated in the streamlined editorial processes and how they supported solution of new problems. It was also essential to shed the light on potential negative implications of the AI expansion for the employment in journalism. Does it really make sense to speak about the actual replacement of the journalist with automated AI systems in the near future?

In the course of our discussions with media experts, understanding of AI was expanded and some automation tools were also assigned to intelligent machine processes.

Despite the fact that the range of the areas, where AI technologies are applied is quite broad (from communication optimization to content distribution management), the article summarizes and highlights those of them, where the progress of interaction is most noticeable.

Analytics to understand and engage the audience. Undoubtedly, the most widely used are the AI analytical tools enabling automatic data processing. They allow avoiding subjective approach to data evaluation. At every stage, obtained results can be verified and adjusted, if necessary. 
The analysis of the user information, its behavior in the media is actively used in targeting tools. AI is able to spot the most popular queries of the audience and be guided by them in the media content generation. However, experts also note a negative feature - a threat of "following the audience" by indulging their content preferences instead of presenting a balanced news agenda.

Content personalization and ranking. Personalized content presentation is a separate area dealing with audience preferences. Applied algorithms allow studying content's user interests to consider them when offering additional content for familiarization. As an example, such initiatives in the video content segment intended to improve viewer engagement are offered in collaboration of IBM Watson Media and Meson by Netflix, which promise users to predict what they want to watch. It should be recognized that digital technologies reoriented television to the affordable, convenient and personalized content with an intuitive user interface and wide adaptation and selection options long ago.

On the websites offering paid access to the content, personalization is also used both to pick up preferred journalist materials, and to determine the scope of user access to various types of media products.

Content generation. "Synthetic media" is the term that has become the buzzword and is currently a driver of many changes primarily effecting the area of news production. Of course, the benefits of the automatic content generation, such as stimulation and democratization of creativity cannot be neglected. AI applied for this purpose supports automatic transformation of the data into various news, images, video and data visualization.

The area of video content shows the greatest involvement in this process. Progress in deep learning that opened the door to the possibility to generate audio and video content is impressive. Editing, which was previously quite complex, has transformed unthinkably. AI can process filmed videos by highlighting the best scenes and takes, and identify content links in dialogs. Being guided by generally accepted rules of composition integrated in the algorithm, the program can autonomously edit media products, while retaining the structure and logic of the narrative. The main character of the video would speak several languages, or may not be a human at all.

So far, the use of AI technologies remains in demand in the segment of audiovisual content production, particularly where it comes to the need to create subtitles for large volumes of materials or archives. At the same time, comparison of traditional overdubbing vs. AI dubbing supported with Synthesia Translate demonstrates astonishing results. Potential of the innovations is obvious for news companies broadcasting in different languages in many regions of the world.

AI voice capabilities have not yet been widely embodied in the media, however, have already proved their usefulness, when for example, they returned on air James Dupree, Cox Media Group, national politics reporter, who lost his voice [11].

Photo banks also improve their photo content-related services and offer journalists efficient instruments for visual content selection. For example, Panel by GettyImage launched in 2018 offers the AI algorithm for analysis of article content and selection of the illustrations that fit the best. However, soon they will probably be unable to compete with fully automated photograph creation processes either.

Even today, resources like Rosebud.AI and Icons8 support generation of photos of non-existent people inscribed in required interiors that may be used as illustrations and substitute photo shoots for publications. According to creators of those photos, in the future, their cost would let avoid referring to photo banks or services of professional photographers. The technology, whose potential was highly appraised by e-commerce, is now conquering the media market. Lil Miquela, a 3D model girl, created by Brud company developers, is known to advertise brands like Calvin Klein, Loreal, "wears" clothes of world-famous brands and has repeatedly appeared on covers of Time, L'Officiel Singapore, Elle Mexico, etc.

All this resolves the issue of routine selection of images for daily content; however, it cannot yet substitute documentary photography, or photography as the art, although the latter can also be implemented with new technologies, but requires presence of a professional.

Search for information and its verification. Despite careful remarks of the interviewed media representatives about use of AI for content generation, journalists are willing to be supported by AI in the information and data processing, which represent a vast amount of work.

Aggregation of news to search for both new topics and specific information facilitates dealing with pools of data indeed. Projects like the Juicer from BBC (that had existed until 2018) perform analysis and group the content according to the set criteria or by category.

Today, the focus of such aggregation has shifted to social networks, which, according to the many, though have not destroyed the media, however, threaten the news journalism. As many events occur in social networks, materials in the genre of "social networks overview" are gaining popularity, whereas the need to optimize the search for information in them is growing. For example, for these purposes, Reuters uses Reuters News Tracer, which according to the company, in terms of the speed often leaves behind world news agencies. Associated Press has its own Newswhip.

AI for data processing and journalist investigations is recognized to be most popular among editorial staff [12]. There are quite many platforms and bots designed to help journalists to check the accuracy of the information; they enable analysis of both the text, visual content and the voiced information [13]. Many of them are the outcomes of the Google News Initiative. At the same time, such resources can be both internal tools facilitating the work of the editorial office, and a mechanism for interaction with the audience and enhancement of its trust. The Poynter Institute's International Fact-Checking Network (IFCN) launches the chatbot project, which everyone can use to check the authenticity of a story.

Knowhere publication, which is a result of human-AI collaboration, may set an example of a new view on what an independent and objective media can be. Its founders call it "the most influential journalist ever built" [14], as the medium reviews a huge number of sources on a specific topic, validates them and based on the outcome, delivers its own unbiased story. 
Data processing and new forms of a live dialog with the audience. Use of machine-supported analytics allowing dealing with big data has found its niche in the sport journalism and coverage of large-scale events with the updatable data. Actually the speed of the sports absorbing and introducing innovations following revolutionary media trends and novel changes that balance on the verge of possible is amazing. [15]

Some time ago, the Washington Post tested processing of the online-received date using intelligent software of Heliograf company. The medium received an award for "Excellence in Use of Bots" for its work on the 2016 election coverage. The Press Association uses Radar for similar tasks of dynamic creation of large amounts of content.

We can say with confidence that chatbot as a simple and easy tool for implementation is becoming increasingly popular in the media industry. Although, commercial companies were first to use it for their 24/7 communication with clients, organization of new formats of a live dialogue with the audience is vital for the media.

Comments, as an essential component of a dialogue with the audience supported by AI, can also be enhanced to allow for live discussions with invited participants and experts. Smart moderation offered by Coral project enables media to involve the audience in real time discussions and manage the dialogue without the fear of destructive comments or undesirable commercials. The Financial Times, the Wall Street Journal, the Washington Post and many other media in 18 countries [16] are extensively using the tool. Perspective API application developed by Jigsaw provides similar options for readers' chats moderation.

However, bots could be used more broadly. One of the options is to integrate a chatbot in journalists' materials as an alternative narrative, which extends the possibilities of a dialogue with readers [17].

Optimization of work processes. Although media representatives admit that they are not ready to accept the format of a fully automated news creation process, they consider the optimization of media production processes to be very useful.

As a rule, such practices (for example, Editor by the New York Times) were closely connected with the trend of convergence editorial office, where all media departments interact with each other and can benefit from the materials being prepared for their structural unit, i.e. put tags, bookmark components of the article, add similar content, or elaborate on the suggested story. This greenlights more efficient multi-purpose use of the obtained information.

By applying Bertie tool to prepare templates of news, Forbes demonstrates that innovations can be used without damaging the profession of a journalist. However, the media insists that substitution of journalists has never been their purpose [18].

Results and discussion. The digital revolution makes an unprecedented impact on the content monetization, creation and dissemination in the media industry. Changes in consumer behavior lead to alterations of business models as well, and make to rethink the competencies required by the industry.

Of course, employment in 4.0 industrialization era experiences severe pressure from global trends in the area of human capital. In the media, the HR strategy is built around data and technologies. HR managers increasingly speak of the need to make a choice in favor of finding talents, encouraging creative thinking and introducing innovations versus let us call them more traditional for journalism work experience, good writing and judgment that were relevant earlier. New technologies force to reconsider sets of skills necessary to remain relevant in the industry. Moreover, the human factor and actually the journalism "with the human face" is still fundamental to the perception of the audience that is deliberately not ready to change it for an impersonal artificially generated news feed. The paradox is that according to the HubSpot's survey, the majority of users (over 63\%) do not realize that they are already using AI technologies [19]. On one hand, this jeopardizes the possibility to identify the owner of the content and evaluate its reliability accordingly, which is especially important today in the midst of the post-truth era and so-called "fake news" epidemic. On the other hand, it gives a chance to the media to build a more active dialogue with the audience without losing the features inherent in the trusted interpersonal communication.

Most of interviewed media representatives agreed that AI would not kill the journalism, and traditional journalist values remain a driving force for them to implement a new work style [20]. Journalists are skeptical about potential fully automated production of news and see no real threat of being substituted by AI. We deliberately will not focus on such examples as Jia Jia robot reporter from Chinese news giant Xinhua, which according to its developers can substantially reduce media production costs, as such robots are not wide spread yet and, in opinion of many respected media analysts, cannot dramatically change the balance of forces of humans vs AI.

When speaking about possibilities to solve new problems provided by AI, we speak of the possibilities for both the audience, and the media themselves. For the audience, they blur the line where digital reality begins and ends, and for the media they reply to the question of how to create high-quality content without the budget and sufficient special professional skills.

As in terms of optimization of editorial and publishing activities media went their own experimental way, currently, many of them have their niche optimization instruments, which apparently will suffer from competition in the next few years with introduction of many similar instruments created with more solid technological and financial resources.

Conclusions. Undoubtedly, AI should become an integral part of the digital media strategy, which would allow getting ahead of industry trends and meeting the audience demands. However, introduction of automated intelligence systems raises many questions, including the intellectual property rights for media products, verification of the information, security, etc.

We highlighted the areas, where use of AI brings the biggest success. Those are data analysis and optimization of work processes, as well as extended interaction with the audience. As to the content generation, journalism takes a conservative position in this regard, and attempts to tell a story completely relying on AI are few. Resources enabling comprehensive verification of the information and treatment of open databases are mainly used by editorial boards focused on analytical materials and journalistic investigations. Observations show that services enabling creation 
and generation of visual content are predominantly used to promote media in social networks. With regard to chatbots, many editors confessed that they did not use them as they lack technical skills necessary to be able to insert them in journalistic narratives. However, chatbots are popular as an instrument of communications with readers concerning subscriptions and donations to the media and ranking search for news (for example, Guardian); media's marketing teams put them in their arsenal too.

Among the constraints, media representatives noted the need for additional investments to implement the AI in work of editorial offices, lack of understanding of all AI benefits and advantages for the media, lack of time and insufficient human resources for refocusing, retraining and obtaining skills for effective use of the AI potential.

In addition, it is obvious that the emergence of new technologies and, consequently, opportunities for the implementation of new tasks, on the contrary, add work in which journalists can be involved and integrated in a large mechanism of a so-called hybrid of a human labor and input of automated systems. Although, almost every activity is stated to have a partial potential for automation, a component of creativity is the buffer mitigating the crisis in the industry and, despite the competition from the automation, journalists would continue to be in demand in their profession.

\section{REFERENCES}

1. New Report 2019 - The next newsroom: unlocking the power of AI for public service journalism. https://www.ebu.ch/news/2019/11/ebu-news-report-urgespublic-service-media-to-work-together-to-face-challenge-ofplatforms.

2. Crespo M. How Artificial Intelligence is Transforming Journalism // Equaltime, 27 November 2018. https://www.equaltimes.org/how-artificial-intelligenceis?lang=en\#.XtElc8Bn02w.

3. Bakker P. Aggregation, Content Farms and Huffinization // Journalism Practice, 2012, 6:5-6, 627637. DOI: 10.1080/17512786.2012.667266;

4. Friedman A. We're all aggregators now. So we should be ethical about it // Columbia Journalism Review, 2014, May 23. https://archives.cjr.org/realtalk/rules_for_ethical_aggregators. php.

5. Stray J. Making Artificial Intelligence Work for Investigative Journalism // Digital Journalism, 2019, 7:8, 10761097. DOI: $10.1080 / 21670811.2019 .1630289$.

6. Singer J. Theorizing Digital Journalism The limits of linearity and the rise of relationships // The Routledge Handbook of Developments in Digital Journalism Studies. London: Routledge, 2019, Chapter 37. DOI: 10.4324/9781315270449.

7. Whittaker J. Tech Giants, Artificial Intelligence, and the Future of Journalism // New York: Routledge, 2019. DOI: 10.4324/9781351013758.

8. Marconi F. Newsmakers: Artificial Intelligence and the Future of Journalism. Columbia University Press, September 2019.

9. Dalen, Arjen Van. The Algorithms Behind the Headlines: how machine-written news redefines the core skills of human journalists // Journalism Practice, 2012, 6(5-6), P. 648-58.

10. Cramer P. Radio - The next frontier for artificial intelligence // Radio Ink, 2017, April 19. https://radioink.com/2017/04/19/radio-next-frontier-artificialintelligence.

11. Reigart E. M. Is artificial intelligence friend or foe to radio? // Radio World.
2018, September 4.
- https://www.radioworld.com/needtoknow/is-artificialintelligence-friend-or-foe-to-radio.

12. Stray J. Making Artificial Intelligence Work for Investigative Journalism // Digital Journalism, 2019, 7:8, P.10761097. DOI: $10.1080 / 21670811.2019 .1630289$.

13. Rothman A. As a Matter of Fact: A New AI Tool for RealTime Fact-Checking of News Using Voice Analysis // The Subway Fold, 2018, December 5. https://subwayfold.com/tag/sparks-grove.

14. Knowherenews.com. https://angel.co/company/knowherehq/jobs.

15. For example: Cormack, J. (2018). The dramatic growth of artificial intelligence in sport // Snack Media, 2018, March 14. - https://www.snack-media.com/2018/03/the-dramaticgrowth-of-artificial-intelligence-in-sport; Galily Y. Artificial intelligence and sports journalism: Is it a sweeping change? // Technology in Society Volume 54, August 2018, P. 47-51.

16. Coral by Vox Media launches new Q\&A tool to support publisher hosted live discussions // Vox Media, 2020, April 24. - https://www.voxmedia.com/2020/4/24/21234360/coral-byvox-media-launches-new-q-a-tool-to-support-publisherhosted-live-discussions.

17. Veglis A., Maniou Th.A. Embedding a chatbot in a news article: design and implementation // 23rd Pan-Hellenic Conference on Informatics (PCI '19). Association for Computing Machinery, New York, 2019, P. 169-172. DOI: $10.1145 / 3368640.3368664$.

18. Zalatimo S. Entering The Next Century With A New Forbes Experience // Forbs, 2018, July 11. https://www.forbes.com/sites/forbesproductgroup/2018/07/11 /entering-the-next-century-with-a-new-forbesexperience/\#425853603bf4 .

19. An M. Artificial Intelligence Is Here - People Just Don't Realize It // HubSpot. https://blog.hubspot.com/marketing/artificial-intelligence-ishere.

20. Diakopoulos N. Automating the News: How Algorithms Are Rewriting the Media. Harvard University Press, 2019, 336 p. 